\title{
EDITORIAL
}

\section{Digital technology in cardiac care}

\section{$\mathrm{T}$} he evolution of digital information technology is progressing rapidly, affecting all aspects of our everyday lives-from how we communicate with others, to our working practices, to how we spend our leisure time. The cultural shift is so great that, although only in widespread use since the 1990s, many of us now wonder how we would manage without the convenience of the internet. The advent of wireless technology and the development of internet-enabled, multimedia 'smart' phones have made the wealth of available information more accessible than ever before. Moreover, the uptake of these devices is astonishing; according to Apple, Inc. (Cupertino, CA), sales of their iPhone ${ }^{\circledR}$ reached 8.7 million in the first fiscal quarter of 2010 alone (http:// www.apple.com/uk/pr/library/2010/01/25results.html). Huge potential exists for the use of digital information technology in the practice of medicine. Currently, several applications exist for these tools in cardiology, including patient education and communication, clinical decision making, improving the speed and efficiency of patient care, automatic detection of abnormal cardiovascular activity in real time, and monitoring physiological variables in patients with coronary conditions.

Patient education and communication between the patient and the physician are vital to the concept of informed consent and this is one area where digital technology has potential application. Research has revealed failings in the traditional system of informed consent across medical disciplines, with patients not fully understanding their condition or the procedures they undergo. Alan Tait and colleagues from the University of Michigan Healthcare System, Ann Arbor, MI and Jackson Memorial Hospital, Miami, FL have demonstrated that interactive, 3-dimensional (3D) software can improve patient understanding of cardiac catheterization before the procedure is performed (Tait, A. R. et al. Arch. Intern. Med. 169, 1907-1914; 2009). The interactive computerized information system produced by ArchieMD ${ }^{\mathrm{TM}}$, Inc. (Boca Raton, FL) utilizes dynamic $2 \mathrm{D}$ and $3 \mathrm{D}$ graphics to guide the patient through a series of modules explaining basic cardiac physiology and the pathophysiology of heart disease, the treatment options available, and full details of the cardiac catheterization procedure. Patients are able to enter questions, which are transmitted to the cardiologist for discussion. At the end of the program, patients take a short quiz to gauge their understanding of the procedure. The program takes just 10-12 min to complete. Individuals who used this system had a greater appreciation of the risks and benefits of cardiac catheterization and alternative treatments than did those who were given standard written

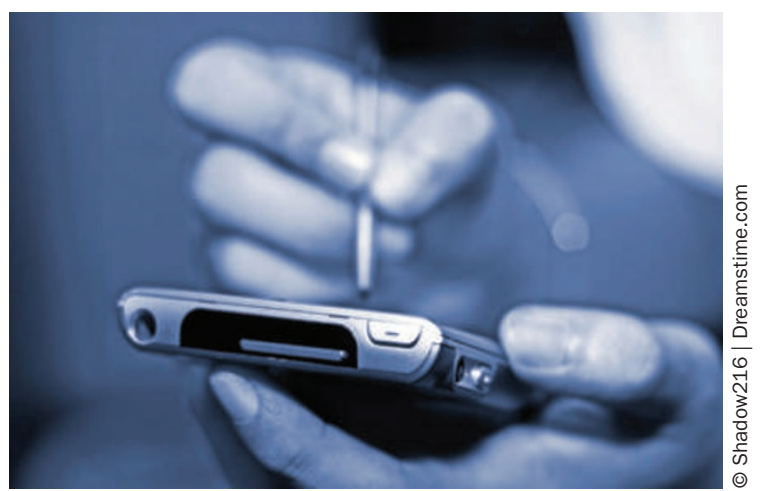

information. Problems with the traditional method of informed consent include lack of patient literacy, language barriers, and the fact that patients often do not understand complex medical terminology. This technology can overcome some of these barriers by being tailored to the individual on the basis of language, literacy, education, and sight-related or hearing-related disability. The interactive nature of the program-with the opportunity to stop and ask questions at any timetogether with the graphical representation of biological systems and procedures, facilitates learning. Although in the early stages of testing, the interactive program by ArchieMD ${ }^{\mathrm{TM}}$, Inc., and other computer-based systems, hold promise for improving patient understanding of complex cardiovascular procedures.

Digital technology can also be used to improve clinical decision making, helping the physician to take a systematic approach toward diagnosis and treatment. Investigators from France have assessed the effectiveness of a hand-held, computer-based decision support system for the evaluation of patients with suspected pulmonary embolism (Roy, P.-M. et al. Ann. Intern. Med. 151, 677-686; 2009). The specially developed software operates on Palm OS $^{\circledR}$ (Palm Computing, Inc., Sunnyvale, CA) smart phones, making access quick and convenient. By entering the patient's clinical characteristics, the physician can obtain a revised Geneva score, which predicts the risk of pulmonary embolism. The program then recommends the most appropriate diagnostic test(s) for the individual patient. The dynamic system allows for reassessment of risk when test results are known, and continues to guide decisions until pulmonary embolism can be confirmed or ruled-out with confidence. In the analysis by Roy and colleagues, physicians who used the decision-support software performed complete and appropriate diagnostic assessment of patients more often than those who used paper-based guidelines. This
Alexandra King is Senior Editor of Nature Reviews Cardiology. Valentin Fuster is the Editor-in-Chief of Nature Reviews Cardiology.

Competing interests The authors declare no competing interests. 
study could, however, be seen to highlight the potential inadequacies of traditional guidelines, rather than the superiority of a computer-based system. Perhaps revising guidelines to make them more comprehensive and userfriendly would result in similar improvements in patient care; only additional research will elucidate this issue. Similar digital decision-support systems have also been used successfully in the screening and management of patients with dislipidemia and to guide anticoagulation regimens. This technology is still in its infancy, but within 3-5 years, we could see the widespread use of such tools across cardiovascular specialties.

One could argue that the widespread use of digital information technology in the diagnosis and monitoring of patients could result in physicians becoming reliant on these tools, losing competency in performing physical examinations and confidence in their own clinical judgment. Clearly, we need to ensure that basic skills, such as good history taking or auscultation, remain as important as ever and will not be lost in the digital revolution (Conn, R. D. \& O'Keefe, J. H. Am. J. Cardiol. 104, 590-595; 2009).

Wireless communication, using products such as Bluetooth $^{\circledast}$ (Bluetooth Sig, Inc. Corporation, Bellevue, WA), can be applied in a multitude of innovative ways to improve the speed and efficiency of patient care. For example, in 2007, Dhruva et al. reported the findings of the STAT-MI (ST-Segment Analysis Using Wireless Technology in Acute Myocardial Infarction) project, which was implemented through the emergency medical services network in the Newark area of New Jersey, USA (Dhruva, V. N. et al. J. Am. Coll. Cardiol. 50, 509-513; 2007). Using Bluetooth ${ }^{\circledast}$-enabled Lifepak ${ }^{\circledast} 12$ defibrillators (Physio-Control, Inc., Redmond, WA) and wireless Motorola ${ }^{\circledR}$ E815 telephones (Motorola, Inc., Schaumburg, IL), emergency services personnel are able to transmit a 12-lead electrocardiogram from a patient with suspected ST-segment elevation myocardial infarction (STEMI) to the emergency room. Once received, the electrocardiogram is automatically converted to PDF format and relayed to the smart phone of the cardiologist on call. Telephone numbers for the emergency team are embedded within the electrocardiogram to facilitate communication with the cardiologist prior to arrival at the hospital. A 6-month trial of this system showed that mean door-to-intervention time was reduced by $66 \mathrm{~min}$ (from $145.6 \mathrm{~min}$ to $80.1 \mathrm{~min}$ ) when compared with data from 2005, before the introduction of the STAT-MI project. Minimizing delays in the treatment of acute STEMI is vital; ACC/AHA guidelines recommend that patients with STEMI should undergo emergency percutaneous coronary intervention within $90 \mathrm{~min}$ of symptom onset (Antman, E. M. et al. J. Am. Coll. Cardiol. 51, 210-247; 2008). More widespread use of such integrated communication systems could go a long way toward achieving this goal.

A similar strategy for monitoring is being developed by interdisciplinary scientists at the University of Pittsburgh (Oresko, J. et al. Proceedings of the Workshop on Biomedicine in Computing: Systems, Architectures, and Circuits 1, 13-16). The HeartToGo ${ }^{\mathrm{TM}}$ system incorporates an electrocardiogram sensor and geographical positioning system (GPS) integrated with the physician's smart phone via Bluetooth ${ }^{\circledast}$. This technology enables automatic detection of abnormal cardiovascular activity in real time. The GPS element allows emergency medical personnel to determine the patient's precise location, thereby potentially cutting response times. The practical application of this technology deserves some thought; continuous monitoring of each patient through a smart phone may not be a realistic option for physicians with a heavy case-load. Risk stratification could be necessary to identify only the most high-risk patients for monitoring. Alternatively, physicians could share monitoring responsibilities-with electrocardiographic information being transmitted to more than one hand-held deviceor trained technicians could monitor information from several patients and relay data to the physician only in the event of an emergency.

Wireless technology also has potential application for monitoring physiological variables in patients with chronic conditions, such as congestive heart failure, or following cardiac intervention. Researchers from Spain and the US have described a novel prototype that combines a wireless microtransmitter with a cardiac pressure sensor and an FDA-approved stent (Chow, E. Y. et al. J. Interv. Cardiol. 22, 479-487; 2009). Chow et al. have tested their device in ex vivo and in vivo models with promising results. Successful wireless transfer of arterial pressures was achieved at an implantation depth of $3.5 \mathrm{~cm}$ with a reception range of $50 \mathrm{~cm}$, thus demonstrating the feasibility of using wireless transmitters within the body. Several other wireless platforms for patient monitoring have been proposed, including an adhesive "smart bandage" that measures respiratory rate, fluid status, heart rate and rhythm, and body temperature in patients with heart failure, and a skin-adhesive patch that can be activated remotely to deliver a set dose of medication (Topol, E. Sci. Transl. Med. 2, 16cm4; 2010).

Clearly, there is no shortage of innovation in the sphere of digital medical technology. The challenge will be to overcome barriers to the quality, efficiency, affordability, accessibility, and safety of these tools. Quality can only be assured through rigorous testing and randomized controlled trials of new technologies, but there must also be a willingness among physicians to perform these studies and among patients to be enrolled in them. The cost of technologies must also be considered and set against potential savings in the cost of health care made by their implementation. Finally, we need to ensure that new innovations are introduced for the right reasons, namely that their accessibility to patients and their safety improve the efficiency of patient management, and overall that the infrastructure exists to allow their widespread use. Let us embrace with open and enquiring minds what could become the 'future' of medicine.

doi:10.1038/nrcardio.2010.26 